\title{
An outbreak of syphilis in Darkhan-Uul, Mongolia, January to March 2012
}

\author{
Battsendiin Munkhzul, ab Batjargaliin Batdorjac and Jantsansengeegiin Baigalmaa ${ }^{a b}$ \\ Correspondence to Battsendiin Munkhzul (e-mail: mzula_mgl@yahoo.com).
}

Background: Fifty-eight syphilis cases were notified from Darkhan-Uul Province in the first three months of 2012, which is double that of the same period in 2011. The objective of this investigation was to confirm the existence of the outbreak, examine the risk factors of cases and develop recommendations to prevent further spread.

Methods: We conducted a descriptive study of cases notified to the national surveillance system diagnosed with syphilis between 1 January and 20 March 2012 in Darkhan-Uul Province. Additional data were collected from doctors' record books and outpatient cards. We compared outbreak cases to non-outbreak cases notified in Darkhan-Uul Province in 2011.

Results: The total notification rate was 62.3 per 100 000. The notification rate was highest among 20-29 year olds, and $38(65.5 \%)$ were females. More males than females were detected through contact tracing (45\% compared with $10.5 \%$ ). Only $10 \%$ of cases reported using condoms during their last sexual encounter, with $65.5 \%$ reporting having had casual sex or multiple sex partners. There were no homosexual cases. Compared to the non-outbreak cases, outbreak cases had a significantly higher proportion of detection through contact tracing (22.4\% compared with $0.7 \%$ ) but a lower proportion of detection through prevention (3.5\% compared with $26.5 \%$ ). There was also a higher proportion of primary syphilis in outbreak cases (62.1\% compared with $41.7 \%$ ).

Conclusion: The syphilis outbreak in Darkhan-Uul Province was confirmed. We recommended effective contact tracing followed by timely diagnosis and treatment. Health promotion activities targeted to high school, college and university students on syphilis infection and prevention as well as condom usage were recommended for future prevention.

S yphilis is a sexually transmitted infection caused by the spirochete bacterium Treponema pallidum. The primary route of transmission is through sexual contact; congenital syphilis can also be transmitted from mother to fetus during pregnancy or at birth. ${ }^{1}$ Blood products can also transmit the infection. ${ }^{1}$ Globally, there are about 448 million new infections of curable sexually transmitted infections (STI) (syphilis, gonorrhoea, chlamydia and trichomoniasis) annually. ${ }^{2}$ In 2010 in Mongolia, there were 14301 cases of STI, representing $34.6 \%$ of all registered infectious diseases. ${ }^{3}$ The proportion of syphilis was $21 \%$ among all reported STI cases in $2010 .^{3}$

Darkhan-Uul Province, located in the northern part of the country, has a population of 93137 . The syphilis notification rate in Darkhan-Uul Province has been higher than national and provincial averages since 2001 to $2009 .^{3}$ Darkhan Health Department reported a twofold increase in syphilis cases in the first three months of 2012 compared to the same period in 2011. Therefore, the aim of this investigation was to confirm the existence of the outbreak, determine the characteristics of cases and develop recommendations to prevent further spread. We also compared cases reported during the outbreak to cases notified in a non-outbreak period in Darkhan-Uul Province.

\section{METHODS}

We conducted a descriptive study of all syphilis cases notified to the national surveillance system from Darkhan-Uul Province between 1 January and 20 March 2012. We included cases diagnosed with any stage of syphilis as classified by STI physicians in accordance with the International Classification of Diseases-10 (ICD-10). ${ }^{4}$ The diagnoses were confirmed using laboratory tests (Rapid Plasma Reagin and Treponema pallidum Hemaglutination Assay).

We collected information from STI outpatient cards and STI doctors' record books for each case. From the record books we collected age, sex, residence, social status, reported detection method and reported source of syphilis infection for a case. Additional information from STI outpatient cards were symptoms and risk

\footnotetext{
Mongolian Field Epidemiology Training Programme, Ulaanbaatar, Mongolia

National Center for Communicable Diseases, Ulaanbaatar, Mongolia.

Khuvsgul Province Health Department, Mongolia.

Submitted: 3 October 2012; Published: 20 December 2012

doi: 10.5365/wpsar.2012.3.4.003
} 
Figure 1. Epidemic curve of syphilis cases reported in Darkhan-Uul Province, Mongolia, January to March 2012 $(n=58)$

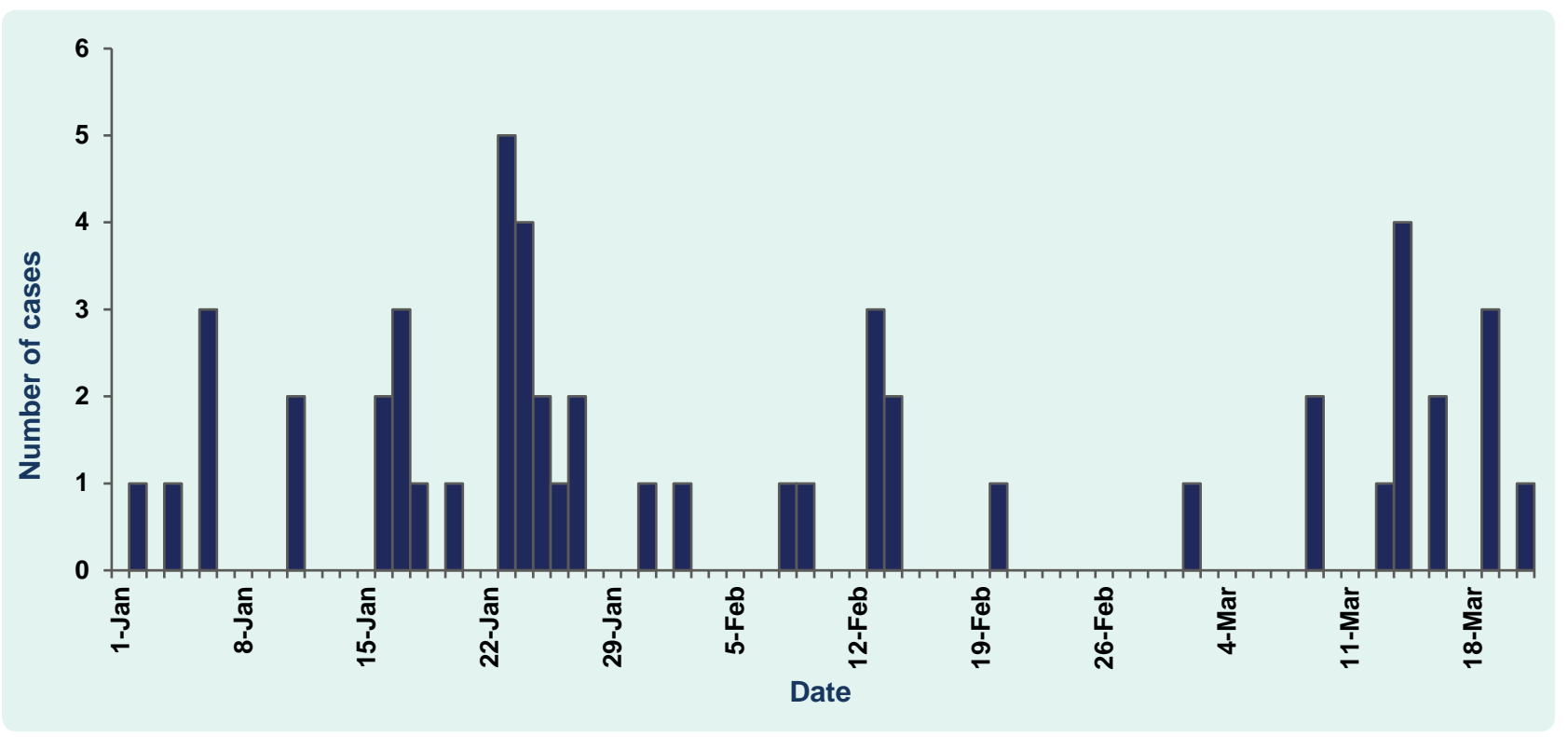

behaviours including: having casual sex (sex with a non-regular sex partner or spouse), condom use during last sexual encounter, having multiple or new sex partners in the last three months, previous STI history, last sexual encounter with whom and where, and condom usage. We compared the characteristics between male and female cases using the chi-squared test in Open-Epi. We compared outbreak cases with nonoutbreak cases reported to the national surveillance system in 2011 from Darkhan-Uul Province. The age distribution, social status, source of infection, detection of infection and stage of syphilis of all outbreak cases to non-outbreak cases were compared using the chisquared test.

We protected the confidentiality of the respondents through the use of codes. Since this work was conducted in the context of an emergency response to an outbreak, it was exempt from ethical clearance.

\section{RESULTS}

There were 58 cases diagnosed with syphilis from 1 January to 20 March 2012 in Darkhan-Uul Province, yielding a notification rate of 62.3 per 100000 . The notification rate for January 2012 was higher than the mean of the number of cases from the same time period in 2011, and it was higher than the threshold of the mean plus two standard deviations. Therefore, this was defined as an outbreak.
Cases were continuously reported between January and March with the highest number of cases reported on 23 January 2012. The number of cases then began to decrease during the early part of February 2012 (Figure 1). There were 38 female and 20 male cases; although the notification rate for females was almost double that for males, this difference was not statistically significant (102.3 per 100000 compared with 54.2 per 100000 respectively, $P=0.4$ ). Median age was 24 years (range: 14-40 years) with the highest incidence among those aged 20-24 (327.5 per 100 000) and 25-29 (207.7 per 100 000) years (Table 1 ).

Addresses were available for 40 cases, and based on residency, there was no obvious geographic clustering of cases; all cases were spread throughout the city. The proportion of cases employed (41.4\%) was higher than those unemployed (17.4\%) and other social groups. There was a higher proportion of employed male cases $(55.5 \%)$ than employed female cases $(34.2 \%)$ but a higher proportion of female students $(26.3 \%)$ compared with male students (15.0\%). However, there was no significant difference in social status of female and male cases $(P=0.2)$ (Table 1$)$.

Most male cases (80.0\%) reported being infected from casual sex, compared with $57.9 \%$ for females. In contrast, $39.5 \%$ of female and $15.0 \%$ of male cases reported being infected by their spouse $(P=0.15)$. Sex workers comprised $15.8 \%(n=6)$ of female cases. 
Table 1. Characteristics of outbreak cases by sex in January to March 2012 and non-outbreak cases in 2011, Darkhan-Uul Province, Mongolia

\begin{tabular}{|c|c|c|c|c|c|c|c|c|}
\hline \multirow{3}{*}{$\begin{array}{l}\text { Characteristics } \\
\text { Age groups }\end{array}$} & \multicolumn{6}{|c|}{ Outbreak cases, 2012} & \multirow{2}{*}{\multicolumn{2}{|c|}{$\begin{array}{c}\text { Non-outbreak cases, } 2011 \\
\text { Total }\end{array}$}} \\
\hline & \multicolumn{2}{|c|}{ Female } & \multicolumn{2}{|c|}{ Male } & \multicolumn{2}{|c|}{ Total } & & \\
\hline & Cases & Rate & Cases & Rate & Cases & Rate & Cases & Rate \\
\hline 14 and below & 1 & 8.7 & 0 & 0.0 & 1 & 4.3 & 0 & 0.0 \\
\hline $15-19$ & 5 & 110.9 & 2 & 36.8 & 7 & 76.7 & 10 & 109.5 \\
\hline $20-24$ & 17 & 327.5 & 5 & 115.3 & 22 & 207.2 & 62 & 583.8 \\
\hline $25-29$ & 9 & 207.7 & 9 & 234.6 & 18 & 207.6 & 37 & 426.8 \\
\hline $30-34$ & 4 & 96.9 & 2 & 55.8 & 6 & 75.4 & 25 & 314.0 \\
\hline $35-39$ & 2 & 51.2 & 1 & 32.1 & 3 & 40.1 & 9 & 120.2 \\
\hline $40-44$ & 0 & 0.0 & 1 & 35.8 & 1 & 14.9 & 3 & 44.8 \\
\hline 45 and above & 0 & 0.0 & 0 & 0.0 & 0 & 0.0 & 5 & 26.2 \\
\hline$P$-value & $P=0.4$ & & & & & & $P=0.3$ & \\
\hline Total & 38 & 102.3 & 20 & 54.2 & 58 & 62.3 & 151 & 162.1 \\
\hline \multicolumn{9}{|c|}{ Section B. Information from STI record book } \\
\hline Social status & Cases & $\%$ & Cases & $\%$ & Cases & Rate & Cases & Rate \\
\hline Children under 15 & 1 & 2.6 & 0 & 0.0 & 1 & 1.7 & 0 & 0 \\
\hline Employed & 13 & 34.2 & 11 & 55.0 & 24 & 41.4 & 58 & 38.4 \\
\hline Unemployed & 7 & 18.4 & 4 & 20.0 & 11 & 19.0 & 45 & 29.8 \\
\hline Sex worker & 6 & 15.8 & 0 & 0.0 & 6 & 10.3 & 5 & 3.3 \\
\hline Other & 1 & 2.6 & 2 & 10.0 & 3 & 5.2 & 5 & 3.3 \\
\hline Student & 10 & 26.3 & 3 & 15.0 & 11 & 22.4 & 38 & 25.2 \\
\hline$P$-value* & $P=0.2$ & & & & & & $P=0.1$ & \\
\hline \multicolumn{9}{|l|}{ Source of infection } \\
\hline Spouse & 15 & 39.5 & 3 & 15.0 & 18 & 31.0 & 69 & 45.7 \\
\hline Sex worker & 1 & 2.6 & 1 & 5.0 & 2 & 3.4 & 1 & 0.7 \\
\hline Casual sex & 22 & 57.9 & 16 & 80.0 & 38 & 65.5 & 81 & 53.6 \\
\hline$P$-value ${ }^{*}$ & $P=0.1$ & & & & & & $P=0.06$ & \\
\hline \multicolumn{9}{|l|}{ Detection of infection } \\
\hline Antenatal care & 12 & 31.6 & 0 & 0.0 & 12 & 20.7 & 27 & 17.9 \\
\hline Due to symptoms & 13 & 34.2 & 11 & 55.0 & 24 & 41.3 & 59 & 39.1 \\
\hline Voluntary visit & 1 & 2.6 & 0 & 0.0 & 1 & 1.7 & 1 & 0.7 \\
\hline Prevention activities & 2 & 5.3 & 0 & 0.0 & 2 & 3.5 & 40 & 26.5 \\
\hline Contact tracing & 4 & 10.5 & 9 & 45.0 & 13 & 22.4 & 1 & 0.7 \\
\hline Second generation surveillance $^{\dagger}$ & 6 & 15.8 & 0 & 0.0 & 6 & 10.3 & 0 & 0 \\
\hline Active dispensary control $^{\ddagger}$ & & & & & & & 23 & 15.2 \\
\hline$P$-value* & $P<0.001$ & & & & & & $P<0.001$ & \\
\hline \multicolumn{9}{|l|}{ Stage of syphilis } \\
\hline Primary syphilis & 21 & 55.2 & 15 & 75.0 & - & - & - & - \\
\hline Secondary syphilis & 12 & 31.6 & 3 & 15.0 & - & - & - & - \\
\hline Latent & 5 & 13.1 & 2 & 10.0 & - & - & - & - \\
\hline$P$-value & $P=0.3$ & & & & & & $P=0.01$ & \\
\hline Total & 38 & 100.0 & 20 & 100.0 & - & - & - & - \\
\hline \multicolumn{9}{|c|}{ Section C. Information from outpatient cards ${ }^{\S}$} \\
\hline \multicolumn{9}{|l|}{ Sexual behaviour } \\
\hline New sex partner in last 3 months & 13 & 52.0 & 13 & 86.7 & 26 & 60.0 & - & - \\
\hline Multiple sex partners in last 3 months & 9 & 36.0 & 10 & 66.7 & 19 & 47.5 & - & - \\
\hline Drank alcohol during or before sex & 3 & 12.0 & 8 & 53.3 & 11 & 27.5 & - & - \\
\hline Received blood or blood product & 1 & 4.0 & 0 & 0.0 & 1 & 2.5 & & \\
\hline Location of sex & & & & & & & & \\
\hline Bar & 0 & 0.0 & 1 & 6.7 & 1 & 2.5 & - & - \\
\hline Hotel & 2 & 8.0 & 8 & 53.3 & 10 & 25.0 & - & - \\
\hline At sexual partner's home & 7 & 28.0 & 1 & 6.7 & 8 & 20.0 & - & - \\
\hline At own home & 15 & 60.0 & 3 & 20.0 & 18 & 45.0 & - & - \\
\hline Outside & 1 & 4.0 & 0 & 0.0 & 1 & 2.5 & - & - \\
\hline Other & 0 & 0.0 & 2 & 13.3 & 2 & 5.0 & - & - \\
\hline$P$-value ${ }^{*}$ & $P=0.002$ & & & & & & & \\
\hline Previous STI history & & & & & & & & \\
\hline Yes & 3 & 12.0 & 3 & 20.0 & 6 & 15.0 & - & - \\
\hline No & 22 & 88.0 & 12 & 80.0 & 34 & 85.0 & - & - \\
\hline$P$-value & $P=0.5$ & & & & & & & \\
\hline Condom usage during last sex & & & & & & & & \\
\hline Yes & 2 & 8.0 & 2 & 13.3 & 4 & 10.0 & - & - \\
\hline No & 23 & 92.0 & 13 & 86.7 & 36 & 90.0 & - & - \\
\hline$P$-value* & $P=0.6$ & & & & & & & \\
\hline Total & 25 & 100.0 & 15 & 100.0 & 40 & 100.0 & - & - \\
\hline
\end{tabular}

* The first $P$-value compares male and female outbreak cases; the second $P$-value compares total outbreak cases and total non-outbreak cases.

The seventh round of second generation surveillance (SGS) among the most-at-risk population represented by female sex workers, mobile men, male STI clients and men who have sex with men was carried out in 2012.

₹ People who are under control of dispensary (mostly female sex workers).

$\S$ In section $\mathrm{C}$, data for 10 cases were not available. 
One third of female cases were identified through antenatal care, another third because they were symptomatic and $10.5 \%$ through contact tracing. In contrast, just over half of male cases were detected because they were symptomatic (55\%) and the rest through contact tracing $(45 \%)$, and these differences were statistically significant $(P<0.001)$ (Table 1$)$.

Among the 40 cases for which outpatient cards were available, $47.5 \%$ reported having had multiple sex partners in the last three months, and 65\% reported having had a new sex partner in last three months. A statistically significant difference in the location of the last sexual encounter was observed between females and males $(P=0.002)$; more than half of male cases $(53.3 \%)$ reported they last had sex at a hotel whereas $60 \%$ of female cases said they had it at home. Only $10 \%$ reported using condoms the last time they had sex. Six cases had a previous history of a STI diagnosis; there was no difference in previous STI diagnoses between females $(12 \%)$ and males $(20 \%)(P=0.5)$ (Table 1).

There were no cases reported from homosexual contact or from injecting drug use.

When the outbreak cases were compared with nonoutbreak cases, outbreak cases had a higher proportion of younger age groups (under 14 and 15-19 year olds) and more sex workers ( $10.3 \%$ compared with $3.3 \%$ ) but a lower proportion of unemployed cases $(19.0 \%$ compared to $29.8 \%$ ). There was a higher proportion of reported casual sex in outbreak cases $(65.5 \%$ compared with $53.6 \%$ ) but a lower proportion of reported infection from spouses (31.0\% compared with $45.7 \%$ ). However, these differences were not significant (Table 1).

There was a significant difference between the two groups for detection $(P<0.001)$ : a higher proportion through contact tracing in the outbreak cases $(22.4 \%$ compared with $0.7 \%$ ) but a lower proportion through prevention activities (3.5\% compared with $26.5 \%$ ). Similarly, there were significant differences for stages of syphilis $(P=0.01)$ : a higher proportion of primary (62.1\% compared with $41.8 \%$ ) and a lower proportion of latent syphilis for outbreak cases ( $12 \%$ compared with $29.8 \%$ ) but similar proportions of secondary syphilis (25.9\% compared with $28.5 \%$ ) (Table 1 ).

\section{DISCUSSION}

We confirmed the syphilis outbreak in Darkhan-Uul Province in early 2012 . Females had higher notification rates than males, and most cases were 20-29 years old. This pattern of more female notifications, which has been observed over the last decade in Mongolia (with the exclusion of 2004 and 2008), ${ }^{3,5}$ is the opposite of patterns reported in China, ${ }^{6}$ Germany ${ }^{7}$ and Sweden, ${ }^{8}$ where male incidence was higher and the majority of male cases were men who have sex with men (MSM). Syphilis outbreaks have also been reported among MSM. $^{9-13}$ Not self-reporting as an MSM in our study may have been due to negative reactions to homosexual people.

In this study, detection of female cases through contact tracing was much lower than male cases. Moreover most male cases were reportedly infected through casual sex whereas the majority of female cases were reportedly infected by their spouse. Possible explanations for this might be that male cases are detected after their spouses' antenatal care visits or that female cases tend to notify their partners more than the male cases. During this outbreak, the proportion of cases detected through contact tracing (22.4\%) was higher than the average proportion of cases detected through contact tracing from reported syphilis cases in Darkhan-Uul Province from 2006 to 2011 at $15.6 \%$.

The lower percentage of unemployed people and the higher percentage of sex workers in the outbreak period could be due to more detection through the National Second Generation STI/HIV Surveillance survey (SGS) conducted in 2012. ${ }^{14}$ More cases are identified through contact tracing in outbreak periods compared to non-outbreak periods. The much higher percentage of symptomatic syphilis identified in this outbreak might be attributable to active contact tracing during the outbreak period. In contrast, the much higher percentage of latent cases identified in the non-outbreak period could be related to identifying cases through prevention examinations that are conducted throughout the year in STI dispensaries.

There were some limitations in this investigation. As the cases were identified through routine surveillance, 
based on ICD-10 case definitions ${ }^{4}$ and Guidelines for STI treatment and services, ${ }^{15}$ some undiagnosed syphilis cases may not have been detected, leading to an underestimation of cases. Also, as we could not link the cases, because the study was conducted from STI record books, we were unable to determine if the cases were related to each other. Our risk factor analysis was limited as the outpatient cards of 18 of 58 cases were not found.

The rate of cases of syphilis in this outbreak was highest in the 15-19 and 20-24 age groups, with $22.4 \%$ of cases being students. The main risk factors for infection in this study were having casual sex and low condom use. Based on these findings, we recommended that contact tracing be improved and guidelines on partner notification services be developed. We also recommended that education, information and communication materials and behavioural change communication activities be targeted to high school, college and university students about STI including syphilis, especially prevention through condom usage.

\section{Conflict of interest}

None declared.

\section{Funding}

This study was undertaken within the Mongolian Field Epidemiology Training Programme (MFETP).

\section{Acknowledgements}

We would like to thank G Surenkhand, vice-director of National Center for Communicable Diseases and director of the MFETP for providing guidance and support. We would like to thank S Uranchimeg, STI physician, and B Erdenetsetseg, epidemiologist, of Darkhan-Uul Health Department for their time and help to complete the analysis. We gratefully thank Luo Dapeng, WHO Mongolia Office; Takaaki Ohyama, Japan-FETP; staff from WHO Regional Office for the Western Pacific who conducted the scientific writing workshop and the MFETP team for their comments and assistance to improve the results of this paper.

\section{References:}

1. Heymann DL. Control of Communicable Diseases Manual, 19th edition, 2008. Washington DC, American Public Health Association, 2008.

2. Sexually transmitted infections - Fact sheet No. 110, August 2011. Geneva, World Health Organization, 2011 (http://www.who.int/ mediacentre/factsheets/fs110/en/, accessed 20 April 2012).

3. Baigalmaa $\mathrm{J}$ et al. Situation of sexually transmitted infections in Mongolia, 2001-2010. Ulaanbaatar, Ministry of Health, 2011.

4. The International Classification of Diseases 10th Edition, Geneva, Word Health Organization, 2010 (http://apps.who.int/ classifications/icd10/browse/2010/en, accessed 10 December 2012).

5. Jantsansengeegiin B et al. Increasing syphilis notifications in Mongolia: results from national surveillance for 2001-2011. Western Pacific Surveillance and Response, 2012, 3(4):86-93. doi:10.5365/wpsar.2012.3.2.008

6. Hesketh T, Ye XJ, Zhu WX. Syphilis in China: the great comeback. Emerging Health Threats Journal, 2008, 1:e6. doi:10.3134/ ehtj.08.006 pmid:22460215

7. Bremer V, Marcus U, Hamouda O. Syphilis on the rise again in Germany - results from surveillance data for 2011, Euro Surveillance: European Communicable Disease Bulletin, 2012, 17(29): $\mathrm{pii}=20222$. pmid:22835467

8. Velicko I, Unemo M. Recent trends in gonorrhea and syphilis epidemiology in Sweden: 2007 to 2011. Euro Surveillance: European Communicable Disease Bulletin, 2012, 17(29): $\mathrm{pii}=20223$. pmid:22835468

9. Yarlagadda $\mathrm{S}$ et al. A syphilis outbreak: recent trends in infectious syphilis in Birmingham, UK, in 2005 and control strategies. International Journal of STD \& AIDS, 2007, 18:410-412. doi:10.1258/095646207781024865 pmid:17609033

10. Hourihan $M$ et al. Lessons from the syphilis outbreak in homosexual men in east London. Sexually Transmitted Infections, 2004, 80:509-511. doi:10.1136/sti.2004.011023 pmid: 15572625

11. Emerson $C R$ et al. The syphilis outbreak in Northern Ireland. International Journal of STD \& AIDS, 2007, 18:413-417. doi:10.1258/095646207781024874 pmid:17609034

12. Cecere D, Senft $S$ and Jones S. Syphilis Outbreak - Alaska, 2011-2012. State of Alaska Epidemiology Bulletin No. 4, 21 February 2012.

13. Cusini $M$ et al. Syphilis outbreak in Milan, Italy. Sexually Transmitted Infections, 2004, 80:154. doi:10.1136/ sti.2003.007526 pmid:15054187

14. Second Generation HIV/STI Surveillance report - 2009. Global Fund Supported Project on AIDS and TB. Ulaanbaatar, Ministry of Health, 2010.

15. Guidelines for STI treatment and services - Order No. 429, dated 10 December 2009. Ulaanbaatar, Ministry of Health, 2009. 